\title{
Fair Quality of Experience (QoE) Measurements Related with Networking Technologies
}

\author{
Isaias Martinez-Yelmo, Isaac Seoane, and Carmen Guerrero \\ Universidad Carlos III de Madrid. \\ Av. Universidad 30. 28911 Leganes (Madrid), Spain \\ \{imyelmo, iseoane, guerrero\}@it.uc3m.es
}

\begin{abstract}
This paper addresses the topic of Fair QoE measurements in networking. The research of new solutions in networking is oriented to improve the user experience. Any application or service can be improved and the deployment of new solutions is mandatory to get the user satisfaction. However, different solutions exist; thus, it is necessary to select the most suitable ones. Nevertheless, this selection is difficult to make since the QoE is subjective and the comparison among different technologies is not trivial. The aim of this paper is to give an overview on how to perform fair QoE measurements to facilitate the study and research of new networking solutions and paradigms. However, previously to address this problem, an overview about how networking affects to the QoE is provided.
\end{abstract}

\section{Introduction}

The first of all, it is necessary to define what QoE means. The concept of QoE can be applied to many topics; therefore, it is necessary to find a wide definition suitable for any field. An interesting, short and concise definition for QoE is: Quality of Experience is a subjective measure of customer's experiences. This definition summarizes the three key points related with QoE. The first one is the fact that QoE is based on measurements, which means some mechanisms are necessary to define what measurements are the most relevant and how they can be obtained. The second one is the subject that takes care of doing the measurements. This subject is the customer/user since it is the person who is paying for a service. This is a very interesting point because it does not matter how difficult is the implementation of a new feature from the user point of view, but the important is how useful are the improvements and benefits that users can experience from their perspective. Finally, it must be taken into account that these measurements are subjective and depend by in the own opinion of each individual person.

The concept of QoE has applicability in many research fields. In fact, the study of QoE is mandatory in any serious deployment related with new products, technologies or processes. 


\section{Evaluation of the QoE}

Internet Service Providers (ISPs) use Quality of Service (QoS) parameters such as bandwidth, delay or jitter to guarantee their users a good service quality. Nowadays, the proliferation of multimedia content makes more important the provision of QoS if a good QoE wants to be provided to the final user. In addition to the classical networking configuration parameters, some advanced streaming techniques exist to minimize the end-to-end loss effects [1] and the links failures despite the guaranteed QoS [2] in the received streaming.

The reception quality with or without the improvements given by these solutions is measured using objective mathematical operations, comparing the original and the received stream, such as packet loss ratio and PSNR (Peak Signal to Noise Ratio). However, this approach has a lack of perceptual quality measurement that should take into account the perception and the understanding of the receiver. Better receiver experience cannot be measured only using good peak values or assuring low mean packet losses because the semantic losses of the received data are not included in these parameters.

At the same time as the multimedia applications spreading, several subjectivity measurement techniques have been studied to address opinion polls. Voice quality has been measured using opinion tests such as MOS (Mean Opinion Score), DRT (Diagnostic Rhyme Test) or DAM (Diagnostic Acceptability Test). This last one was designed to study the effect of voice transmission trough PSTN network and after that to evaluate voice codecs for digital communications.

MOS consists in a number from a simple five-division scale (ACR - Absolute Category Rating Method) [3] that summarizes how the result of a voice transmission or a playout is perceived by people during listening-opinion tests with some common sentences as reference. The numbers used for MOS graduation are illustrated in Table 1(b), The recommendations specify how to perform these tests or how to provide the information to the test subjects. They also explain how to prepare the reference set of a recording speech and the environmental conditions for the tests. All this methodology is specified by ITU-T in [3].

In addition, considering that the multimedia content have been increasing in requirements, some other recommendations has been developed due to the complexity of transcoding audio and video content and its perception by users 4 5 6.7,8. On the other hand, some evaluation tools for video quality measurement have appeared to automatize the opinion scoring [9, 10, to add subjectivity to the traditional frame-to-frame PSNR analysis. These tools, using the original stream as reference, map the opinion of thousand of tests previously made using MOS scoring to well known signal parameter changes to provide subjectivity to end-to-end system quality evaluation.

For statistical evaluation purposes inside the network itself, the Internet Engineering Task Force (IETF) has developed the Media Delivery Index (MDI) that gives an expected value for the video quality based on network parameters, independently of the encoding scheme. The MDI is described by two values: the delay factor (DF) and the media loss rate (MLR). The DF computes how many data units of time of data would need to be buffered to eliminate the jitter and 


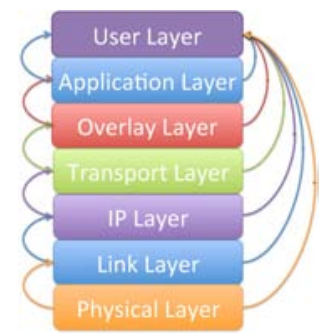

(a) Networking Layers

\begin{tabular}{||c|c||}
\hline \hline Value & Meaning \\
\hline \hline 5 & Perfect \\
\hline 4 & Fair \\
\hline 3 & Annoying \\
\hline 2 & Very annoying \\
\hline 1 & Impossible to communicate \\
\hline \hline
\end{tabular}

(b) MOS score values

Fig. 1.

the MLR is the ratio between the lost and out of order packets and the received packets. Typical maximum acceptable values for MDI depend on the application. They can be $9-50 \mathrm{~ms}$ for DF and from 0.004 on VoD to 0.0005 on HDTV for MLR [1].

\section{Impact of Networking in QoE}

The concept of networking is very wide, in continuous development, and it is always offering new features, advances and improvements. In fact, there are many topics, available technologies and application scenarios related with the networking topic. Thus, it is very difficult to explain how networking can affect the QoE. In order to address this explanation, we follow a layered approach very similar to the current TCP/IP model. Figure 1(a) is an example of this model.

\subsection{Link Layer}

The link layer plays a fundamental role in the user experience, especially in the last mile access. The link layer limits the maximum speed that can be transmitted in a link (Fast Ethernet 1 . In addition, it defines how the information is transported over the links and how the different terminals or computers can access to a link to transmit information.

However, not only these features are provided by the link layer; in addition, the link layer can support Quality of Service (QoS) parameters to prioritize some kind of traffic instead of other ones. This can also be done in Ethernet [12. However, there are not only wired link layer technologies, there are also wireless ones. There is WiFi (802.11) with all its variations, especially the related ones with QoS (802.11e). On the other hand, WiMax (802.16) 13. is also available and can support QoS by default. Obviously, we cannot forget UMTS 14] that provides the wireless infrastructure for $3 \mathrm{G}$ networks.

All previous mentioned technologies (both wired and wireless) are mainly used for shared access, however there are other relevant technologies (usually for

\footnotetext{
1 http://www . ieee802.org/3/
} 
point-to-point communications) such as DSL2 (Digital Subscriber Line) technologies or FTTH (Fiber To The Home) [15, which can also provide QoS.

Although link layer technologies have the same purpose, their speed, capacity, performance and behavior are completely different. Furthermore, their performance can vary depending on the scenario where these technologies are deployed and on any other external conditions. Therefore, it is necessary to describe with the higher accuracy the scenario and external conditions that would be used in a QoE measurement in order to assure the repeatability by other researchers and allow a fair comparison among different technologies. Usually, the most interesting studies about QoE are related with wireless technologies since they are frequently used by end users to retrieve the information from Internet. Some examples are [16], [17].

\subsection{Network Layer}

The network layer is only composed by IP (Internet Protocol [18]). The performance of IP depends on several factors. The first one is the path that packets must follow to reach the desired destination. This path depends on where the end points of a connection are attached, what ISPs give the connectivity to the customers and how the relationships among the different ISPs are. These relationships are usually based on peering and transit agreements among the different ISPs. In order to build correctly the routing tables in the routers of ISPs, BGP (Border Gateway Protocol [19]) is used. BGP manages the Autonomous Systems (ASs), which are a collection of connected IP routing prefixes under the control of one or more ISPs that present a common, clearly defined routing policy to the Internet. Therefore, the behavior of the Internet is based on how the ISPs establish their routing policies through BGP. This fact makes that many research activities related with IP are based on investigation these relationships among ASs and ISPs. There are many projects related with this topic, e.g. CAIDA 3 [20] or PingER [4]. The results from these projects can be used to design and evaluate new proposals in scenarios closer to real conditions. If QoE measurements were considered using the information provided by these projects, the associated measurements would be more realistic and closer to the results in a real deployment.

On the other hand, other important factors affect IP. Originally, IP was designed considering only wired connections where the computers were always placed in the same location. However, with the introduction of wireless technologies and the accessibility to laptops or hand-held devices, the concepts of mobility and roaming arise. Therefore, it is necessary to adapt IP for mobile support: terminal mobility [22,23], network mobility [24] and any other solutions such as multihoming $[25,26$.

In any case, the connectivity among ISPs or the mobility affects delay, jitter and packet error probability and all these factors change the QoE perceived

\footnotetext{
2 http://en.wikipedia.org/wiki/Digital_Subscriber_Line

3 http://www.caida.org/

4 http://www-iepm.slac.stanford.edu/pinger/
} 
by customers. Thus, depending on the scenario and on the adopted technical solutions, the appreciated QoE would be different (e.g. [27,28]).

\subsection{Transport Layer}

The transport layer also implies an important role in the QoE experimented by users. The predominant protocols are TCP (Transmission Control Protocol [29]) and UDP (User Datagram Protocol [30]). TCP plays an important role in best effort bulk transfers because it is a connection-oriented protocol and allows endto-end flow congestion control. However, different congestion control mechanisms can be applied and they affect in a different way the QoE [31]. Besides TCP, other protocols have similar properties and should also be considered such as SCTP (Stream Control Transmission Protocol 32) or DCCP (Datagram Congestion Control Protocol [33]). On the other hand, UDP is a connectionless protocol that usually is used for the transmission of real time information where it is not necessary flow control.

In this section, it is necessary to consider the peer-to-peer traffic that has been growing continuously these last years. A characteristic of peer-to-peer applications is that they need to open many connections (both UDP and TCP) to download the desired resources. These peer-to-peer flows have to compete with the other flows in the Internet. Thus, these last ones are affected negatively by the peer-to-peer traffic. A lot of UDP traffic affects negatively to TCP flows since less bandwidth is available for them. Furthermore, different flows competing for the available bandwidth also implies an underutilization of the total capacity. Therefore, it is necessary to study new mechanisms for the transmission of peer-to-peer traffic in such a way that the QoE experienced by the users of other applications would not be affected. The IETF Low Extra Delay Background Transport (LEDBAT 5 ) Working Group (WG) is taking care about this problem in order to develop lower than best effort transport protocols that avoid to decrease the QoE appreciated in the rest of applications running on the Internet 34].

\subsection{Overlay Layer}

The overlay layer does not exist in the TCP/IP model. However, taking into account the evolution of peer-to-peer technologies, they have to be treated in a single section. A definition of an overlay network can be a computer network that is built on top of another network, usually an IP network. These networks are built at the application layer, but they are in most of the cases an intermediate piece of software that offers functionalities to the final application. Nowadays, the most common overlay networks are the peer-to-peer networks, which are being widely used for file-sharing (eMule [35, 36], Bittorrent 737 ), streaming 8

\footnotetext{
5 http://www.ietf .org/dyn/wg/charter/ledbat-charter.html

6 http://www.emule-project.net

7 http://www.bittorrent.com/

8 http://goalbit.sourceforge.net
} 
and multimedia services (Skype 938,39$]$ ). This kind of applications is not only being considered for these purposes, but they are also being considered for the creation of CDNs (Content Distribution Networks). The adoption of peer-topeer technologies is motivated by moving the traffic consumption of Content Providers (CPs) to their own costumers in order to reduce their payments to their ISPs. However, the peer-to-peer traffic has grown considerably in the last years, affecting users' QoE and overloading the links of ISPs. Some QoE papers related with video streaming and peer-to-peer are [40, 41].

It has been mentioned in the previous section how actions at the transport layer are being studied to reduce their impact. Additionally, actions at this level are also being taken into consideration. The IETF Application-Layer Traffic Optimization (ALTO ${ }^{10}$ ) WG is designing an Application-Layer Traffic Optimization service that will provide applications with information to perform better-thanrandom initial peer selection in order to reduce the traffic between ISPs [42. This service will also help to improve and speed-up the overlay connections of the different peer-to-peer based application. Thus, the QoE experienced by users will improve unless content availability would be compromised (initial seeding). This fact is a remaining open issue.

\subsection{Application Layer}

There are many applications using networking services to work properly. These applications are the only thing that users can really appreciate and interact with. The average customer only takes care of starting an application and using it. In fact, the QoE experienced by customers would not be biased by all the complexity that exists under any application, they only consider if it works or not. An excellent example to consider is Skype 38. Skype was originally a chat and VoIP service based on a peer-to-peer technology. Over the time, it has been improving its functionalities in order to get more and more customers. Nevertheless, the great success of Skype is not based on its functionalities, but on the fact that you install the software in any computer and network configuration and works without any complex configuration (install and use). This simplicity for the users is based on its extraordinary capabilities of crossing NATs and firewalls without reconfiguring any network equipment. Thus, in order to realize fair QoE measurements it is necessary to use the same networking conditions to compare different applications or use the most close conditions if different networking technologies are being compared. An example about QoE related with Skype is 43].

\subsection{Cross-Layer Interactions}

Although protocol design is usually based on a layered approach (for simplicity considerations), it must be taken into account that cross-layer interactions exist.

\footnotetext{
${ }^{9}$ http://www.skype.com

10 http://www . ietf.org/dyn/wg/charter/alto-charter.html
} 
These interactions can affect considerably the QoE experienced by customers. It is well known the effect of wireless technologies to the TCP throughput since losses in wireless link are misinterpreted by network congestion in TCP. Some efforts are being realized to minimize these cross-layer interactions (e.g. the IEEE 802.2111 initiative). Thus, it is necessary to specify the conditions that have been adopted in any application or new networking technology since scenarios that seem to be quite similar can make obtain unexpected results. This fact can prevent the fair comparison of different solutions and lead to wrong conclusions. In the next paragraphs, we are going to detail a methodology in order to prevent this kind of situations. Some studies related with this topic and QoE are 44, 45]

\section{Fair QoE Measurements in Networking}

Taking into account the description given in the previous sections, we can appreciate the great number of variables that it is necessary to take into account when the QoE has to be evaluated. Therefore, it is necessary to follow a methodology that avoids unfair comparisons among different solutions, which would end in wrong conclusions.

Nowadays, the QoE is usually related with the Content Distribution across the Internet. Years ago, it was not considered the use of Internet for establishing voice or video calls and it was also not considered its usage for video broadcasting to a determined group of users. These new services are the focus of CPs and ISPs to get more profits from their networks. Therefore, it is necessary a mechanism to evaluate and compare VoIP and video services or new services such as CDNs.

The proposal that is going to be described in the next paragraphs has been developed inside the CONTENT NoE (see section 5 for further information). This proposal is motivated by the lack of well-defined metrics to compare and evaluate this kind of solutions. The idea is to define a Functional Benchmarking 12 (FBM) framework that facilitates fair comparisons among different proposals. This framework can be used for quantitative measurements such as Content Delivery or search efficiency (something important in CDNs) but also qualitative measurements related with QoE. The interesting point of this approach is the effort spent to normalize how to specify the experiments performed in order to allow a posterior fair comparison with all the research community.

\subsection{Functional Benchmarking (FBM) Framework}

The objective of this framework is to define a methodology that allows the evaluation and comparison of different solutions in a systematic and organized way. This approach tries to define a fair mechanism to compare different proposals. A comparison is said to be fair if the conditions that have been used in the different experiments are exactly the same. Therefore, the FBM is capable of defining all the external variables that affect an experiment in order to assure its posterior

\footnotetext{
11 http://www.ieee802.org/21/

12 http://heim.ifi.uio.no/ plageman/Site_3/Benchmarking.html
} 
repeatability. This fact implies the definition of the environment, the workload that has been used to stress the system under evaluation and what information has been retrieved. Furthermore, it is necessary to specify precisely what metrics have been used to measure the different parameters. In the next paragraphs, we explain the different concepts in the FBM.

\subsection{System Under Test (SUT)}

This framework defines an entity named System under Test (SUT). This entity is formed by a set of functions that want to be evaluated using this methodology. An example could be the QoE experienced by a user with a mobile phone that is receiving a video stream under certain mobility conditions.

\subsection{Input Parameters}

These parameters define conditions used for the evaluation of the SUT. These conditions have a direct impact in the system that is being evaluated and they define the results that would be obtained. In this framework, the input parameters are divided in two different classes:

- Environment. It defines the computational and communication infrastructure as well as other relevant conditions for the assessment. The environment reflects the static conditions of the system under evaluation.

- Workload. It defines the parameters that are used to evaluate the SUT. The workload reflects the dynamic conditions of the system under evaluation.

The importance of this classification is to distinguish among the conditions that are imposed by the experiment itself (environment) and those conditions that stress the SUT (workload). For instance, the environment is related with the access technology used in the experiment, the OSs (Operative Systems) where the experiments are being executed or the selected peer-to-peer network for the development of an application. On the other hand, the workload is related with the background traffic in the different links, the number of entities participating in the experiment or the mobility pattern of the terminals that are being used in a certain testbed.

Following with the example presented in the definition of SUT, the environment would be any characteristic that belongs to the system under study. In that case, the mobile terminal could have a $3 \mathrm{G}$ connection with multihoming support. The workload would be external conditions to the system that affect to the perceived quality such as the concurrent users using the $3 \mathrm{G}$ cell.

\subsection{Output Parameters}

These parameters define the results obtained from the SUT when it has been tested under certain environment and workload. These parameters are also divided in two classes: 
- Performance. The parameters in this class are related with the obtained efficiency in the SUT.

- Cost. This class if formed by those parameters that reflect the necessary resources used to achieve the obtained performance.

This classification helps to separate two different types of results that are usually considered. The results associated with the quality/performance of SUT and, on the other hand, the cost needed to achieve that quality/performance. A solution can obtain an exceptional performance t but it can consume too many resources, which is not desirable.

Again, if we consider the scenario in the definition of SUT, we can define the performance and cost parameters for that example. The performance associated to QoE would be the score given by users to the quality of the streamed videos. On the other hand, the cost would be the amount of traffic used to obtain that performance.

\subsection{Using FBM}

An example of using the FBM could be as follows: a new VoIP application based Chord Peer-to-Peer has to be analyzed on a wireless and mobile IP scenario.

The idea of using the FBM is to facilitate as much as possible the test environment to allow a fair comparison among different future proposals. The result of applying the FBM is shown in Table 1 This table indicates the different input parameters that must be specified to assure the repeatability of the experiments and the relevant output parameters that should be considered to compare the different proposals. The table is divided considering the different layers that have been mentioned previously. In addition, a user layer is included to introduce the QoE related measurements. In the considered example, a MOS score for the VoIP conversations should be provided by the users of the application using an environment and workload with the specific values that should be provided with each one of the parameters considered in table 1. In the proposed scenario, it must be taken into account the underlying network topology, background traffic, number of wireless stations competing for the channel access, etc. The quality of a VoIP conversation will not be the same when the scenario is composed by two stations belonging to the same wireless LAN rather than two wireless stations connected through several ASs. Therefore, it is necessary to specify this

Table 1. QoE measurements with FBM

\begin{tabular}{|c|c|c|c|c|}
\hline \multirow{2}{*}{ Layer } & \multicolumn{2}{|c|}{ Input } & \multicolumn{2}{c|}{ Output } \\
\cline { 2 - 5 } & Environment & Workload & Performance & Cost \\
\hline User & screen size,light & - & MOS & - \\
\hline Application & Codec & Load CPU, RAM & Decoding Time & CPU, RAM consumption \\
\hline Overlay & Chord & N peers & Delay, hops & Bandwidth consumption \\
\hline Transport & UDP & Concurrent flows & Packet Losses/Errors & Overhead \\
\hline Network & Mobile IP,Topology & N handoffs,traffi & Packet Losses & N hops,Delay \\
\hline Link & 802.11 & N stations,traffi & Availability,Retransmissions & Energy, Delay \\
\hline Physical & Noise & N interferences & - & - \\
\hline
\end{tabular}


information. Furthermore, it must be taken into account the type of peer-to-peer network in order to allow the comparison with other topologies in the future.

\section{Conclusions}

This papers presents a short overview on how the QoE can be measured and how the different layers in a networking environment can influence in the perceived QoE. Furthermore, it presents different works related with QoE and the different layers in a networking environment. Finally, in order to facilitate a fair comparison among different research proposals, a Functional Benchmarking (FBM) Framework is defined to specify the necessary information to fairly compare different proposals (this framework is a joint research in the Content NoE). This framework establishes the definition of an environment and workload at the different layers that defines the state of the SUT. Furthermore, it specifies the output parameters that measure the efficiency and cost of the different proposals under evaluation. For each experiment must be defined with high precision any parameter to assure the complete repeatability of the experiments. The proposed framework helps to specify these parameters in an ordered and meaningful way.

\section{Acknowledgments}

We would like to acknowledge Marco Gramaglia for his comments. This work has been funded by the CONTENT NoE from the European Commission (FP62005-IST-41) and by the Ministry of Science and Innovation under the CONPARTE project (MEC, TEC2007-67966-C03-03/TCM) and T2C2 project grant (TIN2008-06739-C04-01).

\section{References}

1. Chakareski, J., Han, S., Girod, B.: Layered coding vs. multiple descriptions for video streaming over multiple paths. Multimedia Systems 10(4), 275-285 (2005)

2. Begen, A.C., Altunbasak, Y., Ergun, O., Ammar, M.H.: Multi-path selection for multiple description video streaming over overlay networks. Signal Processing: Image Communication 20(1), 39-60 (2005)

3. ITU-T p.800 methods for subjective determination of transmission quality - series p: Telephone transmission quality; methods for objective and subjective assessment of quality. Technical report, ITU-T (August 1996)

4. Takahashi, A., Hands, D., Barriac, V.: Standardization activities in the ITU for a QoE assessmen of IPTV. IEEE Communications Magazine 46(2), 78-84 (2008)

5. ITU-T rec. p.910: Subjective video quality assessment methods for multimedia applications. Technical report, ITU-T (April 2008)

6. ITU-T rec. bt.500-12: Methodology for the subjective assessment of the quality of television pictures. Technical report, ITU-T (September 2009)

7. ITU-T rec. bt.710: Subjective assessment methods for image quality in highdefinition television. Technical report, ITU-T (November 1998) 
8. ITU-T rec. bt.1788: Methodology for the subjective assessment of video quality in multimedia applications. Technical report, ITU-T (January 2007)

9. Pinson, M., Wolf, S.: A new standardized method for objectively measuring video quality. IEEE Transactions on broadcasting 50(3), 312-322 (2004)

10. van den Branden Lambrecht, C., Verscheure, O.: Perceptual Quality Measure using a Spatio-Temporal Model of the Human Visual System. In: Proc. IST/SPIE Conference Digital Video and Compression: Algorithms and Technologies, vol. 2668 (1996)

11. IPTV QoE: Understanding and interpreting MDI values. Technical report, Agilent Technologies (2006)

12. IEEE: 802.1Q-2005. IEEE Standard for Local and Metropolitan Area NetworksVirtual Bridged Local Area Networks (2005)

13. IEEE: 802.16-2009. IEEE Standard for Local and metropolitan area networks Part 16: Air Interface for Broadband Wireless Access Systems (2009)

14. 3gpp: 3gpp Release 9 (December 2009)

15. Nowak, D., Murphy, J.: FTTH: The overview of existing technologies. In: SPIE Opto-Ireland 2005: Optoelectronics, Photonic Devices, and Optical Networks, Citeseer, vol. 5825, pp. 500-509 (2005)

16. Piamrat, K., Viho, C., Bonnin, J.M., Ksentini, A.: Quality of experience measurements for video streaming over wireless networks, pp. 1184-1189 (April 2009)

17. Piamrat, K., Ksentini, A., Bonnin, J.M., Viho, C.: Q-dram: Qoe-based dynamic rate adaptation mechanism for multicast in wireless networks, pp. 1-6 (December 30-April 2009)

18. Postel, J.: Internet Protocol. RFC 0791, Internet Engineering Task Force (September 1981)

19. Rekhter, Y., Li, T., Hares, S.: A Border Gateway Protocol 4 (BGP-4). RFC 4271, Internet Engineering Task Force (January 2006)

20. Claffy, K., Hyun, Y., Keys, K., Fomenkov, M., Krioukov, D.: Internet mapping: From art to science. In: CATCH '09: Proceedings of the 2009 Cybersecurity Applications \& Technology Conference for Homeland Security, Washington, DC, USA, pp. 205-211. IEEE Computer Society, Los Alamitos (2009)

21. Cottrell, L., Satti, F., McKee, S., Kalim, U.: (icfa-scic) 2010 report. Technical report, International Committee for Future Accelerators - Standing Committee on Inter-Regional Connectivity (2010)

22. Perkins, C.: IP Mobility Support for IPv4. RFC 3344, Internet Engineering Task Force (August 2002)

23. Johnson, D., Perkins, C., Arkko, J.: Mobility Support in IPv6. RFC 3775, Internet Engineering Task Force (June 2004)

24. Devarapalli, V., Wakikawa, R., Petrescu, A., Thubert, P.: Network Mobility (NEMO) Basic Support Protocol. RFC 3963, Internet Engineering Task Force (January 2005)

25. Dhraief, A., Montavont, N.: Toward mobility and multihoming unification: the shim6 protocol: a case study. In: Wireless Communications and Networking Conference, WCNC 2008, pp. 2840-2845. IEEE, Los Alamitos (2008)

26. Bagnulo, M., Garcia-Martinez, A., Azcorra, A.: Ipv6 multihoming support in the mobile internet. IEEE Wireless Communications 14(5), 92-98 (2007)

27. Soto, I., Bernardos, C., Calderon, M., Banchs, A., Azcorra, A.: Nemo-enabled localized mobility support for internet access in automotive scenarios. IEEE Communications Magazine 47(5), 152-159 (2009)

28. Bernardo, F., Vucevic, N., Umbert, A., Lopez-Benitez, M.: Quality of experience evaluation under qos-aware mobility mechanisms, pp. 1-7 (June 2008) 
29. Postel, J.: Transmission Control Protocol. RFC 0793, Internet Engineering Task Force (September 1981)

30. Postel, J.: User Datagram Protocol. RFC 0768, Internet Engineering Task Force (August 1980)

31. Trinh, T., Sonkoly, B., Molnár, S.: On the fairness characteristics of fast tcp. Annals of Telecommunications 65(1), 73-85 (2010)

32. Stewart, R.: Stream Control Transmission Protocol. RFC 4960 (Proposed Standard) (September 2007)

33. Kohler, E., Handley, M., Floyd, S.: Datagram Congestion Control Protocol (DCCP). RFC 4340 (Proposed Standard) Updated by RFCs 5595, 5596 (March 2006)

34. Shalunov, S.: datagram congestion control protocol, Internet Draf draft-ietf-ledbatcongestion-01.txt (March 2010)

35. Brunner, R., Biersack, E.: A performance evaluation of the Kad-protocol. Technical report, Corporate Communications Department. Institut Eurécom (2006)

36. Steiner, M., En Najjary, T., Biersack, E.W.: Analyzing peer behavior in KAD. Technical Report EURECOM+2358, Institut Eurecom, France (October 2007)

37. Erman, D., Ilie, D., Popescu, A.: Bittorrent session characteristics and models. Traffic Engineering, Performance Evaluation Studies and Tools for Heterogeneous Networks 61(84), 61

38. Baset, S.A., Schulzrinne, H.G.: An analysis of the skype peer-to-peer internet telephony protocol. In: INFOCOM 2006. 25th IEEE International Conference on Computer Communications. Proceedings, April 2006, pp. 1-11 (2006)

39. Rossi, D., Melia, M., Meo, M.: A detailed measurment of skype network traffic. In: IPTPS 2008 (2008)

40. Lu, Y., Fallica, B., Kuipers, F.A., Kooij, R.E., Mieghem, P.V.: Assessing the quality of experience of sopcast. Int. J. Internet Protoc. Technol. 4(1), 11-23 (2009)

41. da Silva, A., Rodriguez-Bocca, P., Rubino, G.: Optimal quality-of-experience design for a p2p multi-source video streaming, pp. 22-26 (May 2008)

42. Xie, H., Yang, Y.R., Krishnamurthy, A., Liu, Y.G., Silberschatz, A.: P4p: provider portal for applications. SIGCOMM Comput. Commun. Rev. 38(4), 351-362 (2008)

43. Huang, T.Y., Huang, P., Chen, K.T., Wang, P.J.: Can Skype be more satisfying? - a QoE-centric study of the FEC mechanism in the internet-scale VoIP system. IEEE Network (2010)

44. Tsagkaropoulos, M., Politis, I., Dagiuklas, T., Kotsopoulos, S.: Enhanced vertical handover based on 802.21 framework for real-time video streaming. In: Mobimedia '09: Proceedings of the 5th International ICST Mobile Multimedia Communications Conference, pp. 1-6. ICST, Brussels (2009)

45. Andersson, K., Granlund, D., Ahlund, C.: M4: multimedia mobility manager: a seamless mobility management architecture supporting multimedia applications. In: MUM '07: Proceedings of the 6th international conference on Mobile and ubiquitous multimedia, pp. 6-13. ACM, New York (2007) 